\title{
CEMENTO BIOCERÁMICO COMO BARRERA APICAL EN DIENTE PERMANENTE NO VITAL CON ÁPICE INMADURO
}

\author{
Bioceramic cement as an apical barrier in a permanent non-vital tooth with an \\ immature apex
}

\author{
Sarmiento-Cárdenas Andrea ${ }^{1}$, Gutiérrez-Pulla Karen ${ }^{2 *}$, Bastidas-Calva Zulay ${ }^{3}$. \\ ${ }^{1}$ Estudiante de 10mo ciclo de la Carrera de Odontología, Universidad Católica de Cuenca. Ecuador \\ 2 Odontóloga de consulta particular Cuenca. Ecuador \\ ${ }^{3}$ Docente de la Carrera de Odontología, Universidad Católica de Cuenca. Ecuador \\ *karen-tefy@hotmail.com
}

\begin{abstract}
Resumen
La terapia endodóntica de los dientes con ápices inmaduros asociados a diversas patologías es la apexificación. Desde hace muchos años los materiales convencionales utilizados en esta técnica fueron el Hidróxido de Calcio y MTA. Es así, que debido a los varios limitantes que estos presentan en sus indicaciones clínicas se creó el Biodentine, con propiedades físico mecánicas superiores semejantes a la dentina natural, que estimula a la cicatrización de tejidos perirradiculares dañados y regeneración ósea. En este reporte de caso clínico se muestra un tratamiento exitoso de apexificación a los 6 meses, tras un traumatismo dentoalveolar y presencia de periodontitis apical crónica, a base de un cemento de trisilicato de calcio como sello apical, lo que comprueba que este material también puede remplazar a la dentina a nivel radicular.
\end{abstract}

Palabras clave: Cemento de silicato, Periodontitis apical, apexificación.

\begin{abstract}
The endodontic therapy of teeth with immature apices associated with various pathologies is apexification. For many years the conventional materials used in this technique have been Calcium Hydroxide and MTA. Thus, due to the several limitations that these materials present in their clinical indications, Biodentine was created, with superior physical-mechanical properties similar to natural dentine, which stimulates the healing of damaged periradicular tissues and bone regeneration. This clinical case report shows a successful apexification treatment at 6 months, after dentoalveolar trauma and presence of chronic apical periodontitis, based on a calcium trisilicate cement as apical seal, which proves that this material can also replace dentine at root level.
\end{abstract}

Key words: Silicate cement, periapical periodontitis, apexification.

\section{Introducción}

Los dientes permanentes jóvenes son susceptibles al daño pulpar ocasionado por caries, lesiones endoperiodontales, traumatismos, exposición pulpar mecánica, tratamiento ortodóntico o anomalías congénitas que conducen a la pérdida de la vitalidad y desarrollo radicular incompleto. ${ }^{1-5}$ Esto a su vez, resulta en la formación de raíces cortas con paredes dentinarias muy delgadas que aumentan la fragilidad del órgano dentario y en la falta de una constricción apical natural que dificulta la terapia endodóntica, ya que la presencia de un foramen abierto no proporciona una barrera anatómica. ${ }^{1-5}$ Radiográficamente, también se puede evidenciar que un diente con estas características clínicas presenta un amplio espacio del conducto radicular. ${ }^{5}$

Durante décadas, los dientes incompletamente formados han sido tratados mediante la apexificación. ${ }^{1-6}$ La Asociación Americana de Endodoncia AAE la define como "un método que induce la formación de una barrera calcificada en un diente con ápice abierto o la continuación del desarrollo apical de una raíz incompletamente formada en dientes con pulpa necrótica con la finalidad de inducir o permitir la formación de una barrera calcificada que oblitere adecuadamente el orificio apical o que permita el desarrollo radicular completo permitiendo el posterior tratamiento 
endodóntico convencional, facilitando así un buen sellado hermético del sistema tridimensional de conductos", 4,7-11

Esta técnica fue descrita por Alfred L. Frank en el año 1966, inicia con el desbridamiento o eliminación completa del tejido necrótico, luego se procede con la desinfección de conductos y colocación intraradicular del hidróxido de calcio, con recambios cada 3 a 6 meses hasta que se pueda evidenciar, mediante un estudio radiográfico, la cicatrización de la lesión apical presente, formación de la barrera con tejido calcificado y un completo desarrollo radicular, prosiguiendo finalmente con la obturación de los conductos. . $^{1,3,5,6,9,10}$

A pesar de su potente función antimicrobiana, este método ha reportado grandes desventajas, como la formación de un barrera mineralizada porosa e irregular, mayor riesgo a la fractura radicular a nivel cervical (debido a que la aplicación del hidróxido de calcio durante periodos prolongados debilita la estructura del diente), sellado y ajuste pobre, no permite la aposición de dentina en las paredes del conducto, no favorece al aumento de la longitud radicular del diente y la duración del tratamiento que va de 6 a 24 meses requiriendo de varias citas. ${ }^{1,3-5,7-10}$

Esto condujo a modificaciones en los procedimientos de la apexificación tradicional, como la obturación del canal radicular mediante la creación de una barrera apical con agregado de trióxido mineral MTA sin necesidad de cierre completo apical. ${ }^{3-5,8-11}$ El MTA es un cemento a base de silicato de calcio, que debido a sus características físicas y químicas, como excelente biocompatibilidad, buena capacidad de sellado, baja solubilidad, mayor radiopacidad que la dentina y buena adaptación marginal se ha convertido en el material de elección para la apexificación con tapón o plug apical, también se emplea en casos de recubrimiento pulpar directo, pulpotomías obturaciones retrógradas, perforaciones radiculares, reabsorciones internas y externas. ${ }^{1,4,7-14}$

De acuerdo con Torabinejad y Chivian el procedimiento clínico consiste en dos sesiones. ${ }^{1,10,14}$ En la primera, iniciamos con anestesia, aislamiento absoluto, preparación de un acceso adecuado, desinfección con clorhexidina de los conductos y la colocación de hidróxido de calcio durante 7 a 14 días. ${ }^{1,10,11,14}$ En la segunda, se retira la medicación intraconducto con abundante irrigación, secamos con conos de papel estéril, preparamos el MTA mezclando el polvo con agua estéril y con el porta amalgama o messing gun llevamos la mezcla a los conductos, con la ayuda de los condensadores apicales se condensa suavemente creando el tapón apical de 3 a $4 \mathrm{~mm}$, verificamos su extensión mediante una radiografía periapical, colocamos una bolita de algodón humedecida en el conducto y sellamos con material provisorio la cavidad. ${ }^{1,10,11,14}$ Luego de 4 horas retiramos la restauración provisional y procedemos a obturar con gutapercha los conductos, sellamos la cavidad de acceso y finalmente realizamos la restauración definitiva. ${ }^{1,10,14}$

Esta técnica presenta grandes ventajas, como la finalización del tratamiento en un menor número de citas, obturación en dos sesiones a diferencia del hidróxido de calcio, efecto antibacteriano, capacidad de inducir a la formación de cemento o tejido duro y de ligamento periodontal lo que lo convierte en un material biológicamente aceptable para el cierre apical. ${ }^{1,5,7-11,14}$ Sin embargo, a pesar de su eficacia, el MTA requiere de un tiempo de fraguado largo, tiene un alto costo, dificultad en su manejo clínico, puede provocar decoloración en la estructura dental y al igual que el hidróxido de calcio no estimula el desarrollo radicular. ${ }^{3,4,9-11}$

\section{Reporte de caso}

Paciente de sexo masculino de 19 años de edad, con aparente buen estado de salud general, acudió a la Clínica Docente de Odontología de la Universidad Católica de Cuenca, al motivo de consulta respondió "me duele el diente izquierdo". Al realizar el examen clínico intraoral se observaron piezas dentarias con ausencia de caries, sin embargo, la pieza dentaria 2.6 presentaba una restauración provisional. Al test de frío con Cloruro de Etilo la respuesta fue negativa, siendo positiva a la prueba de percusión vertical refiriendo dolor moderado.

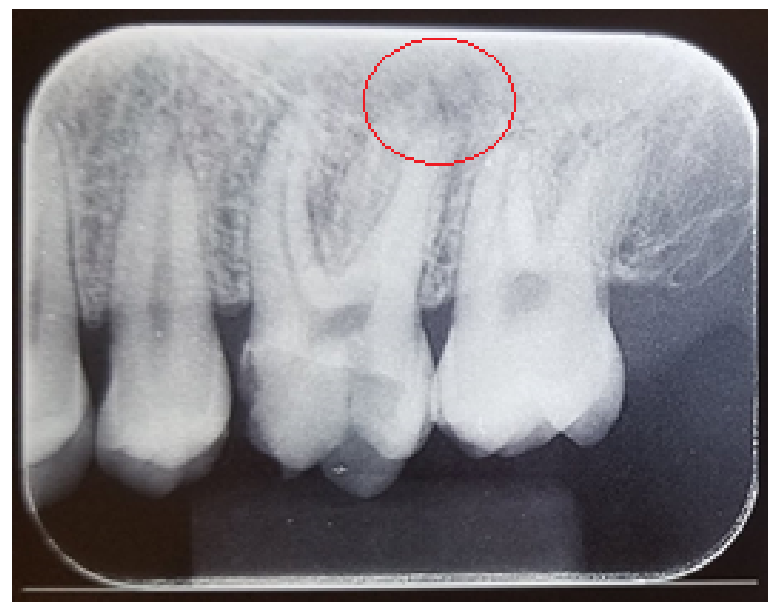

Fig. 1. Radiografía periapical de la pieza dentaria 2.6: cavidad cariosa con compromiso a nivel de la cámara pulpar, sombra radiolúcida a nivel apical de todas las raíces, ensanchamiento del ligamento periodontal y ápice abierto en raíz palatina.

Tras la aplicación de pruebas térmicas de sensibilidad pulpar, de percusión y el análisis del examen radiográfico, se estableció el diagnóstico de periodontitis apical sintomática. 
El paciente con previo conocimiento de los posibles riesgos y complicaciones, accedió al plan de tratamiento que consistió en una fase inicial endodóntica con necropulpectomía II y apexificación, y una segunda fase de rehabilitación oral mediante la colocación de poste, tallado y corona:

\section{Acceso coronal y desgaste compensatorio}

Luego del aislamiento absoluto, se inició con el acceso endodóntico en forma trapezoidal, logrando localizar cuatro conductos: mesiovestibular 1 (MV1), mesiovestibular 2 (MV2), distovestibular (DV) y palatino.

\section{Conductometría}

A través del localizador de foramen se obtuvo la longitud de trabajo para cada conducto: MV1 $19.5 \mathrm{~mm}$, MV2 $18.5 \mathrm{~mm}$, DV $21 \mathrm{~mm}$ y $20 \mathrm{~mm}$ en el conducto palatino.

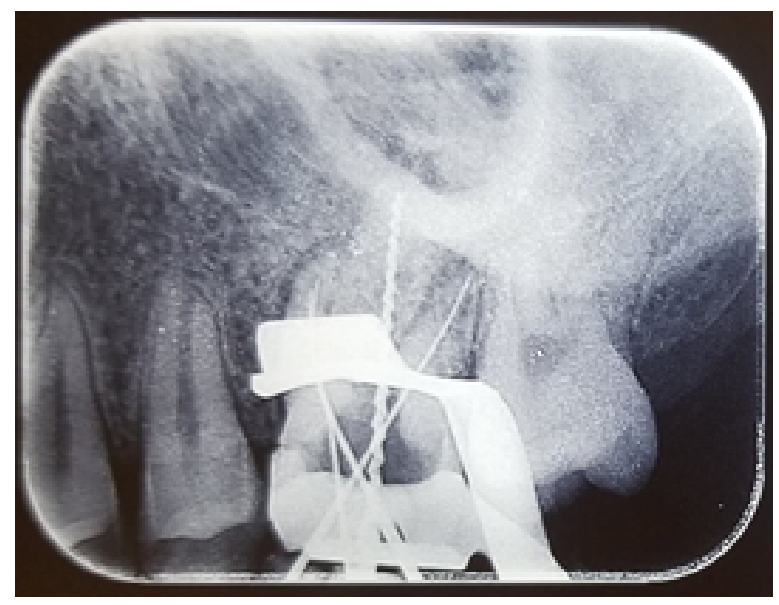

Fig. 2. Radiografía periapical de la pieza dentaria 2.6: uso de lima $\mathrm{K}$ $\mathrm{N}^{\circ} 25$ en el conducto MV1, lima $\mathrm{K} \mathrm{N}^{\circ} 20$ en el conducto MV2, lima $\mathrm{K} \mathrm{N}^{\circ} 20$ en el conducto DV y lima $\mathrm{H} \mathrm{N}^{\circ} 60$ en el conducto palatino. Obsérvese la pieza dentaria 2.5 con ápice abierto.

Luego de observar el ajuste de la lima $\mathrm{H} \mathrm{N}^{\circ} 60$ en el conducto palatino y corroborar con el localizador de ápice, mismo que al emitir su sonido revelaba un foramen aparentemente abierto, se solicitó realizar una CBCT en la que se evidenció la falta de cierre apical a nivel de la raíz palatina y una amplia zona radiolúcida que también comprometía las raíces vestibulares del diente a tratar.

Preparación biomecánica de los conductos radiculares

En la segunda cita, empleando la técnica corono-apical manual, se procedió con la preparación biomecánica con limas $\mathrm{K}$ e irrigación de hipoclorito de sodio al $5 \%$ con una aguja Navitip 30G de $25 \mathrm{~mm}$ (color azul). Al concluir este procedimiento, se colocó la medicación intraconducto a base de hidróxido de calcio dejándola actuar por 8 días. La pieza dentaria fue restaurada provisionalmente con coltosol.

En la tercera cita se retiró el hidróxido de calcio, se neutralizó y nuevamente lo aplicamos por 8 días más.
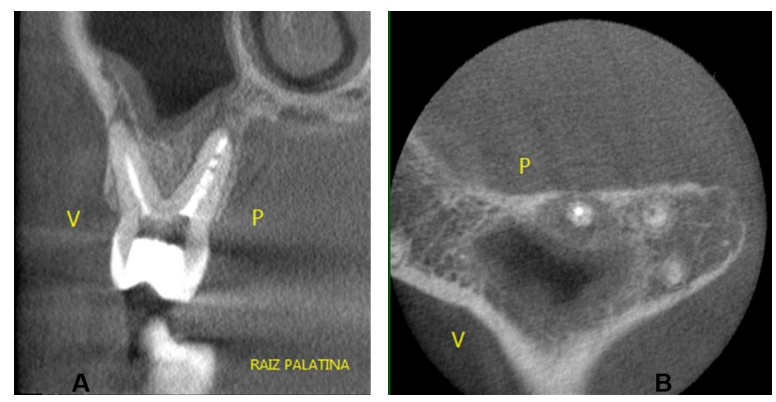

Fig. 3. CBCT de la pieza dentaria 2.6. A) Corte coronal: posterior a la aplicación de medicación intraconducto con Hidróxido de Calcio. B) Corte axial: amplia lesión periapical a nivel del conducto palatino.

\section{Conometría}

En la cuarta cita se realizó la conometría de los conductos MV1, MV2 y DV.

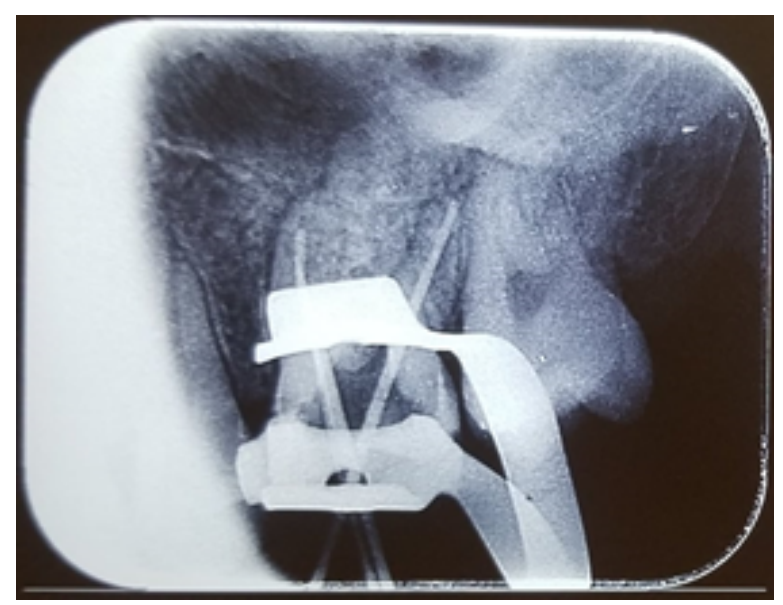

Fig. 4. Radiografía periapical de la pieza dentaria 2.6: conos principales $\mathrm{N}^{\circ} 50$ en el conducto MV1, $\mathrm{N}^{\circ} 25$ en el conducto MV2 y $\mathrm{N}^{\circ}$ 50 en el conducto DV.

En esta misma sesión se continuó con los procedimientos descritos a continuación:

\section{Barrera apical con Biodentine}

Al abrir la cápsula del polvo de Biodentine colocamos 5 gotas del líquido contenido en la pipeta (misma que debe ser golpeada levemente con la finalidad de que todo 
el líquido descienda), cerramos la cápsula y la colocamos en el amalgamador (a una velocidad de 4000 a 4200 oscilaciones por minuto). Se mezcló durante 30 segundos y nuevamente, se abrió la cápsula para comprobar si la consistencia obtenida era de tipo macillosa-densa. Con el porta amalgama se tomó el material y con un condensador tipo Matchou Plugger con tope de goma, calibrado a $16 \mathrm{~mm}$ se colocó el cemento en el tercio apical, formando un tapón apical de una longitud aproximada de $4 \mathrm{~mm}$. Esperando los 12 minutos correspondientes de fraguado total para este cemento biocerámico.

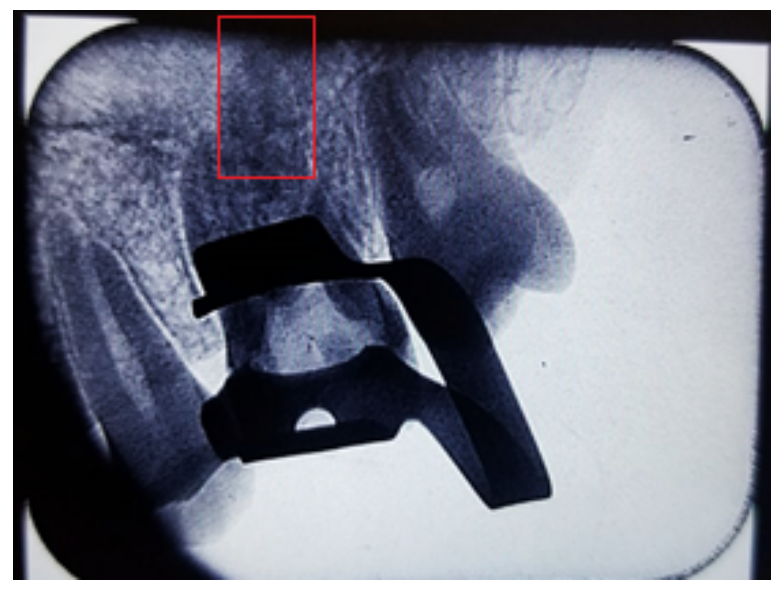

Fig. 5. Radiografía periapical de la pieza dentaria 2.6: tapón apical en el conducto palatino con Biodentine. Baja radiopacidad del material.

La obturación de los conductos restantes se realizó con la técnica de condensación lateral. Luego de irrigar con hipoclorito de sodio al $2.5 \%$ y secar los conductos con conos de papel, se preparó el cemento sellador Sealapex. Los conos principales fueron empapados con este cemento y colocados en los conductos correspondientes, así el cono $\mathrm{N}^{\mathrm{o}} 50$ en el conducto MV1, el $\mathrm{N}^{\mathrm{o}} 25$ en el conducto MV2 y el $\mathrm{N}^{\circ} 50$ en el conducto DV; se añadieron también conos accesorios con la ayuda de espaciadores y condensadores Matchou Plugger color gris punta $\mathrm{N}^{\circ} 3$ para la condensación vertical. Los excesos fueron retirados con el cortador de gutapercha. La pieza dentaria fue restaurada provisionalmente con ionómero de vidrio de restauración.

\section{Rehabilitación oral con corona metal-cerámica}

En la quinta cita, a los 15 días de la aplicación de la barrera apical con Biodentine, en el conducto palatino se colocó un poste de fibra de vidrio con fines protésicos. La pieza dentaria fue restaurada con composite, continuando esta con su respectivo tallado.

Luego de la toma de impresiones definitivas y una vez obtenida la corona metal-cerámica, previa comprobación de

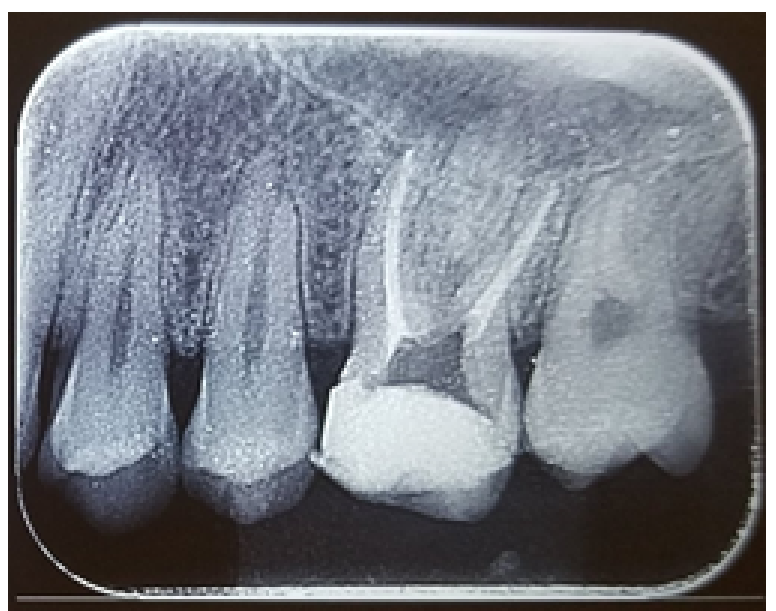

Fig. 6. Radiografía periapical de la pieza dentaria 2.6: Obturación del conducto MV1, MV2 y D.

una correcta adaptación y ausencia de problemas oclusales, articulares y musculares se procedió a su instalación.
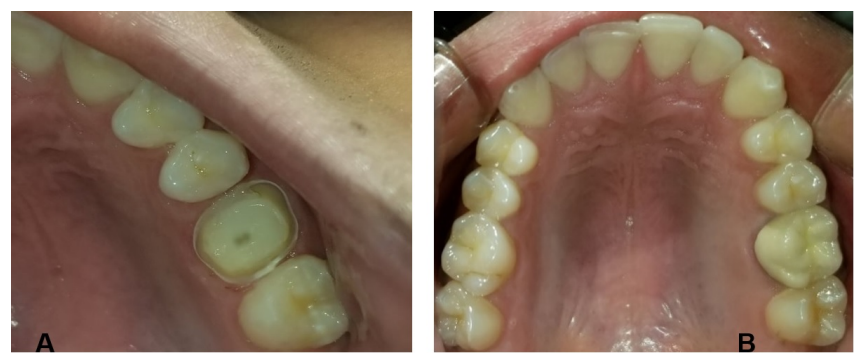

Fig. 7. A) Fotografía intraoral lateral izquierda: tallado yuxtagingival, desmineralización a nivel distal de la pieza 2.6. B) Fotografía intraoral del maxilar superior: cementación de la corona metalcerámica en la pieza dentaria 2.6, finalizando la rehabilitación total del diente.
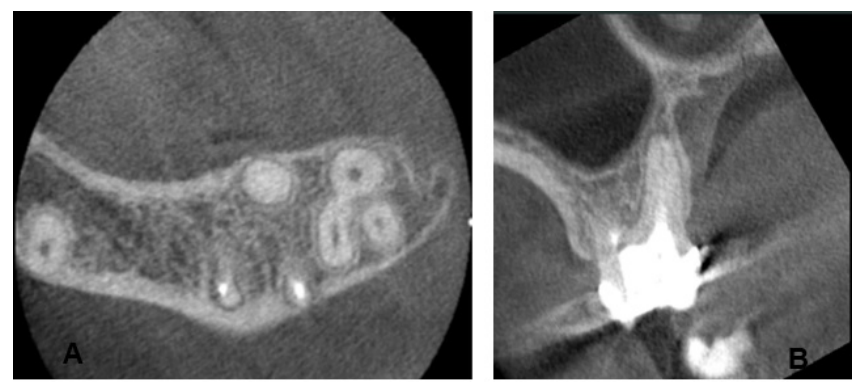

Fig. 8. CBCT de la pieza dentaria 2.6. A) Corte axial: reparación ósea a nivel apical de la raíz palatina, tomografía a los 6 meses de control. B) Corte longitudinal: reparación ósea de los tejidos periapicales a los 6 meses de colocado el tapón apical con Biodentine en la raíz palatina. 


\section{Controles}

Se solicitó al paciente acudir a controles periódicos a los 15 días, 6 meses y al año. En el primer control no se evidenció sintomatología clínica asociada al tratamiento rehabilitador. En el segundo control y con el objetivo de examinar radiográficamente solicitamos una CBCT, con la que verificamos la disminución de la lesión apical, reparación de los tejidos periapicales y especialmente la formación de tejido óseo.

\section{Discusión}

En los últimos años, con el objetivo de crear un material a base de silicato de calcio, con propiedades físico-mecánicas superiores a las del $\mathrm{Ca}(\mathrm{OH}) 2$ y MTA, se desarrolló el cemento Biodentine. ${ }^{9,12-14}$ Está compuesto por una fase en polvo de silicato tricálcico (regula la reacción de fraguado), carbonato de calcio (actúa como relleno) y dióxido de zirconio (otorga radiopacidad), y una fase líquida constituida de cloruro de calcio (acelera el fraguado), agua y polímero hidrosoluble (reduce el agua); elementos minerales, que brindan pureza al producto. ${ }^{8,9,12,13}$

Este material, presenta propiedades que ofrecen grandes ventajas dentro del campo endodóntico. ${ }^{8,13}$ Por su propiedad bioactiva, similar a la dentina, posee un alto pontencial de cicatrización y reparación de tejidos periapicales, induce a la fomación de dentina terciaria, promueve la regeneración del complejo dentino pulpar y conserva su vitalidad. $8,9,12,13$

Según estudios realizados, el Biodentine aumenta la secreción de TGF- $\beta 1$ (factor de crecimiento transformador) de las celulas pulpares y la síntesis de BMP-2 (proteína morfogénica ósea) escenciales para la diferenciación odontoblástica y osteoblástica, por consiguiente, son responsables de la dentinogésis reparativa. ${ }^{8,13,15-17}$ Esto lo convierte en el material indicado para sustituir la dentina en procedimientos de apexificación, restauraciones definitivas y temporales, recubrimiento pulpar, pulpotomías, reparaciónes de perforaciones radiculares y furca, reparación de reabsorciones, obturación a retro y regeneración endodóntica, sin evidenciar signos posteriores de inflamación o sensibilidad. . $^{8,12,13,15-19}$

A su vez, las óptimas propiedades físicas y mecánicas proporcionan adecuadas condiciones para conservar la vitalidad pulpar y lograr la durabilidad de las restauraciones, gracias a su hermeticidad a nivel dentinario, mayor resistencia mecánica (semejante a la dentina) por el bajo grado de porosidad, excelente adaptación, mejor resistencia a la tracción, buen sellado marginal, densidad aumentada, vizcosidad, corto tiempo de fraguado (12 min), obturación en una sola sesión y coloración estable, superando las desventajas del $\mathrm{Ca}(\mathrm{OH}) 2$ y MTA. ${ }^{8,9,12,13,15-19}$ Sin embargo, su principal desventaja es su baja radiopacidad..$^{8,12,17,19}$

En cuanto a su propiedad de biocompatibilidad, las pruebas biológicas no han demostrado efectos citotóxicos, mutagénicos o irritantes por lo que es seguro para el uso odontológico al aplicarlo directamente sobre el tejido, este cemento no causa daños en las células pulpares y no contiene monómero; siendo comparable al MTA.8,9,12,13,15-18 De igual manera, su acción antibacteriana crea un ambiente desfavorable para los microorganismos, esto se debe, al incremento del pH y concentración de iónes de calcio que mejoran las características de esta propiedad. ${ }^{12,13,17}$

No obstante, existen ciertas limitaciones en su manejo, como en casos de restauraciones con gran pérdida de estructura dentaria, tratamiento de pulpitis irreversible y restauraciones estéticas. ${ }^{15}$ Así como también, el empleo de presión excesiva en el proceso de condensación o un recorte exagerado pueden alterar los componentes del producto, perdiendo este su dureza. ${ }^{12}$

La información presentada demuestra un avanzando enfoque terapéutico del Biodentine para solucionar la problemática endodóntica, especialmente en el caso clínico reportado en este artículo describimos los resultados al emplearlo en una terapia de apexificación convencional, los mismos que coinciden con los obtenidos por los siguientes autores:

Shiha y cols., reportaron el manejo de una lesión periapical a causa de terapia endodóntica fallida. ${ }^{20}$ De acuerdo a los hallazgos clínicos y radiográficos, eligieron un enfoque conservador como línea de tratamiento. ${ }^{20}$ El protocolo empleado incluyó instrumentación mínima, desinfección alternada del conducto y pasta tripleantibiótica por dos semanas. ${ }^{20}$ En ausencia de síntomas, colocaron un tapón de $5 \mathrm{~mm}$ de Biodentine en el tercio apical, esperando 12 minutos de fraguado para sellar el resto del canal con gutapercha termoplastificada. ${ }^{20}$ Sus seguimientos clínicos evidenciaron, después de 3 semanas, ausencia de sintomatología y de tracto sinusal, a los 12 meses a través de una CBCT, la reducción total de la lesión periapical y buena cicatrización de los tejidos, con presencia de una barrera calcificada a nivel apical. ${ }^{20}$ Por su parte, Khetarpal y cols., también destacan el uso de Biodentine en los casos de lesiones periapicales grandes debido a traumatismo dental, pues tras realizar controles del procedimiento a los 3 y 18 meses, observaron ausencia de síntomas y de tracto sinusal, regeneración completa de tejidos perirradiculares y la formación de hueso. ${ }^{21}$ Es de importancia mencionar que pese a lograr con éxito el cierre apical, al igual que el resto de cementos silicatos, el Biodentine no promovió el aumento de la longitud radicular. ${ }^{7,9,12}$ 


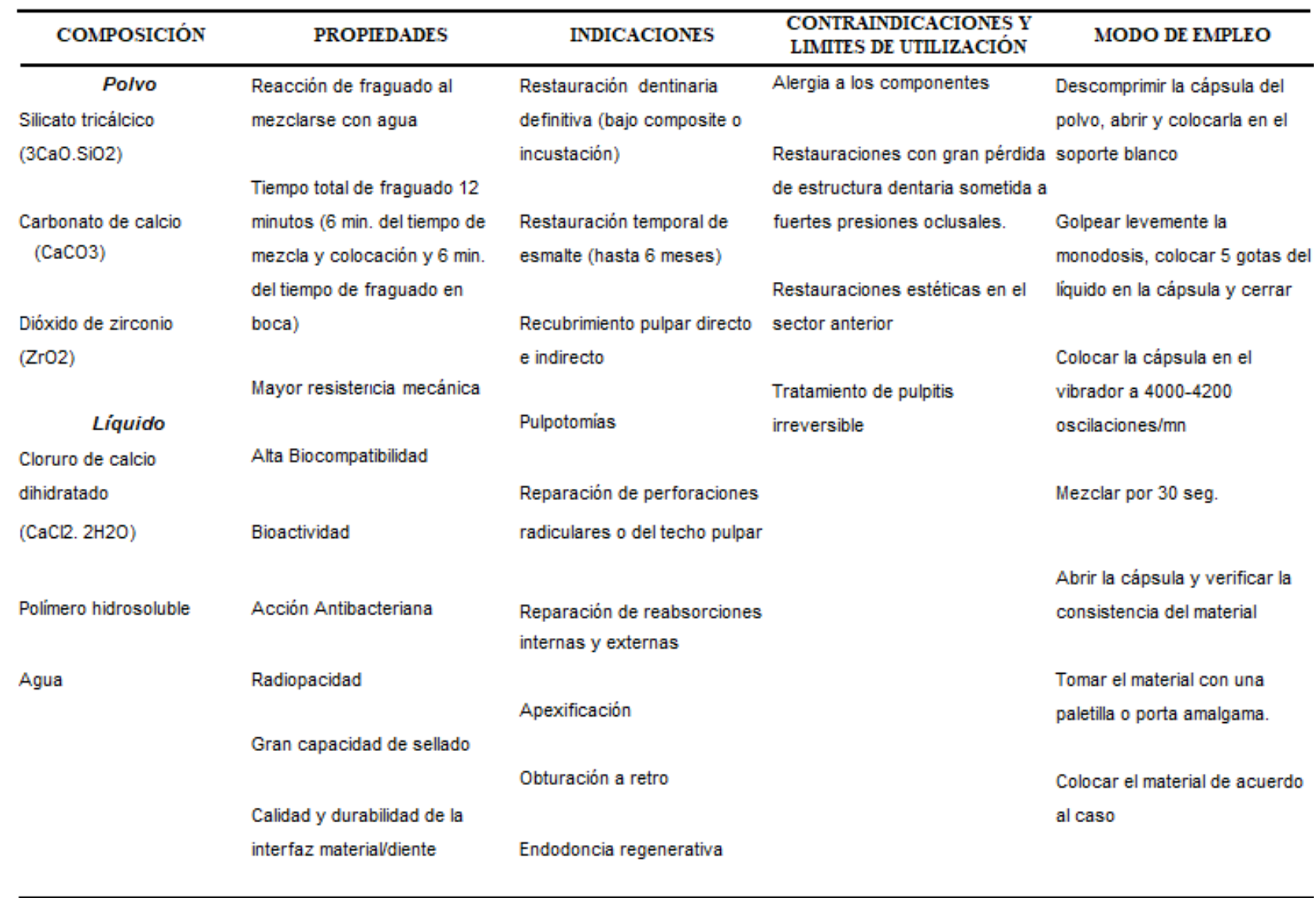

Tabla 1. Principales caracteristicas y alternativas clínicas del Biodentine en las complicaciones de la terapia endodóntica. ${ }^{8,9,12,13,15,16,17,18,19}$

\section{Conclusiones}

Concluimos entonces, que debido a sus propiedades mejoradas este material en comparación con el $\mathrm{Ca}(\mathrm{OH}) 2$ y MTA es la mejor alternativa como matriz de barrera apical y que el éxito del tratamiento se basa en la elección adecuada de la terapia radicular, un minucioso protocolo de desinfección de conductos y de su precisa colocación a nivel apical. No obstante, la literatura actual sobre la aplicación de esta terapia en casos clínicos como el expuesto es escasa, por lo que se recomienda continuar con más investigaciones.

Agradecimiento: Los autores agradecen al Dr. Diego Palacios Vivar (Departamento de Investigación, Carrera de Odontología, Universidad Católica de Cuenca) por su ayuda en la revisión de este manuscrito.

Conflictos de interés: Los autores declaran que no tienen ningún conflicto de interés.

\section{Referencias}

1 Mohammadi Z. Strategies to manage permanent non-vital teeth with open apices: a clinical Update. Int Dent J. 2011; 61(1): 25-30.

2 Fernández D. Nuevas tendencias de andamios en los procedimientos regenerativos endodónticos (tesis de especialización en Endodoncia). Ciudad de Bárbula. Universidad de Carabobo. 2019.

3 Regis M. Conceptos actuales en el tratamiento de dientes jóvenes con necrosis pulpar (tesis de especialización en Endodoncia). Ciudad de Mendoza. Universidad Nacional de Cuyo. 2017.

4 Hernández C, Guerrero M, Gutiérrez I, Corona A. Apexificación utilizando el hidróxido de calcio como primera alternativa de tratamiento. Odontol Pediatr. 2015; 14(2): 150-157.

5 Coaguila H, Denegri A. Uso de barreras apicales y apexificación en endodoncia. Rev Estomatol Herediana. 2014; 24(2):120-126.

6 Frank A. Therapy for the divergent pulpless tooth by continued apical formation. JADA. 1966; 72:87-93. 
7 Songtrakul K, Azarpajouh T, Malek M, Sigurdsson A, 21 Khetarpal A, Chaudhary S, Talwar S, Verma M. Endodontic Kahler B, Lin L. Modified Apexification Procedure for Immature Permanent Teeth with a Necrotic Pulp/Apical Periodontitis: A Case Series. JOE. 2020; 46(1): 116-123.

8 Vidal K, Martin G, Lozano O, Salas M, Trigueros J, Aguilar G. Apical Closure in Apexification: A Review and Case Report of Apexification Treatment of an Immature Permanent Tooth with Biodentine. J Endod. 2016; 42(5): 730-734.

9 Cardoso A, Herrera D, Laurindo A, Nagata J, Rocha T, Soares A. Alternativas clínicas para el tratamiento de dientes traumatizados con rizogénesis incompleta: una visión actualizada. Rev Estomatol Herediana. 2016; 26(4): 271280.

10 Umashetty G, Patil B, Rao N, Ajgaonkar N. Apical Closure of Nonvital Permanent Teeth: 15 Months Follow-up Study of Four Cases. Journal of International Oral Health. 2015; 7(5):71-73.

11 Bidar M, Disfani R, Gharagozloo S, Khoynezhad S, Rouhani A. Medication with Calcium Hydroxide Improved marginal adaptation of mineral trioxide aggregate apical barrier. $\mathrm{J}$ Endod. 2010; 36(10): 1679-1682.

12 Cedrés C, Giani A, Laborde J. Una Nueva Alternativa Biocompatible: BIODENTINE. Actas Odontol. 2014; 11(1): 11-16.

13 Hincapié S, Valerio A. Biodentine: Un nuevo material en terapia pulpar. Univ Odontol. 2015; 34(73): 69-76.

14 Velásquez V, Álvarez M. Tratamiento pulpar en la apexificación del diente inmaduro mediante agregado de trióxido mineral. Odontol Sanmarquina. 2009; 12(1): 29-32.

15 Bachoo I, Seymour D, Bruton P. A biocompatible and bioactive replacement for dentine: is this a reality? The properties and uses of a novel calcium-based cement. British Dental Journal. 2013; 214(5): 1-7.

16 Nikfarjam F, Beyer K, Konig A, Hofmann M, Butting M, Valesky E et al. Influence of Biodentine $₫$ - A Dentine Substitute - On Collagen Type I Synthesis in Pulp Fibroblasts In Vitro. PLOS ONE. 2016; 11(12): 1-20.

17 Ameneiros O, Gamboa J, Soto A, Martínez A, Ruiz H. El uso de materiales bioactivos en la estomatología conservadora contemporánea. Biodentine $®$. Invest Medicoquir. 2019; 11(3): 1-14.

18 Véliz V, Tapia P. Resistencia a la tracción de postes de fibra de vidrio cementados en dientes con perforaciones radiculares selladas con Biodentine ${ }^{\circledR}$ y Proroot ${ }^{\circledR}$ Mta. Rev SECH Canal Abierto. 2019; 39: 4-9.

19 Corral C, Fernández E, Martín J, Estay J, Bersezio C, Cisternas $\mathrm{P}$ et al. Revisión del estado actual de cementos de silicato de calcio en odontología restauradora. Rev Fac Odontol Univ Antioq. 2016; 27(2): 425-441.

20 Sinha N, Singh B, Patil S. Cone beam-computed topographic evaluation of a central incisor with an open apex and a failed root canal treatment using one-step apexification with Biodentine ${ }^{\mathrm{TM}}$ : A case report. J Conserv Dent. 2014; 17(3): 285-289. management of open apex using Biodentine as a novel apical matrix. Indian J Dent Res. 2014; 25(4): 513-516.

Recibido: 02 de junio de 2020

Aceptado: 01 de octubre de 2020 
